\title{
The Importance of Immunology Knowledge for the Health Sciences Students and Physicians
}

\author{
Marcano-Lozada M1,2* \\ ${ }^{1}$ Aggregate Professor Microbiology Department, "J.M. Vargas" School of Medicine, \\ Universidad Central de Venezuela, Caracas, Venezuela \\ ${ }^{2}$ Medical Microbiology Specialist, Medical Microbiology Unit, Angios Vascular Center \\ \& Wound Clinic, Caracas, Venezuela
}

\section{Commentary}

Volume 1 Issue 2

Received Date: November 11, 2019

Published Date: November 22, 2019

DOI: $10.23880 /$ aii-16000108

*Corresponding author: Marcano-Lozada M, Department of Microbiology, JM Vargas School of Medicine, Central University of Venezuela, Caracas, Venezuela, Email: marcelmarcano@gmail.com

\section{Commentary}

When I was a sophomore medicine student, I had the first real contact with immunology, the second most fear subject of the second year of the med studies (behind physiology), but I never suspect the importance of this subject in my formation as a doctor and in the day-to-day practice in general medicine and then as a Medical Microbiology Specialist.

For the medical student, the immunology knowledge is basic to understand surrounded subjects like microbiology \& physiology, and for the next years of the medical formation, the direct relationship with pathophysiology, pharmacology, infectious diseases, pediatrics \& internal medicine is vital to understand the knowledge of all of them. In many occasions, we really appreciate the importance of this world of knowledge when we have the opportunity to find it merge in a pathological situation, in a diagnostic test, etc., outside the isolated field of the basic immunology, so, I'm a true believer that the immunology knowledge for health science students and physicians should be basic, but mainly applicated immunology, because we're not formal laboratory scientist! We're the ones who stay at patients' bedside.

I want to tell you some examples for real-life situations take from my personal experience (from so many years ago!), when I was studied the complement system, I can believe how this amazing cascade of different factors could participate in the defense against bacteria (mostly capsulated), and increase the immune response in a positive way to eliminate that infection... but, as I previously told you, I really understand the importance of this immunological knowledge when I studied microbiology and it consolidates when I began to attend to the clinical rounds and work with patients with immunodeficiency's (more susceptible to be infected by some kind of bacteria).

In a similar way to the previous experience, when I faced a patient with human immunodeficiency virus infection, the T-cell depletion becomes it in a perfect target to specific infections (bacterial, mycotic, parasitic, viral), because the main cell of the defensive system is in inoperative mode by the action of the HIV, in other words, immune-cell mediated defense against infections is crush. In addition, in my private practice, I had the opportunity to watch the effects of the immune reconstitution syndrome with the use of drug cocktails in the HAART (Highly Active Anti-Retroviral Therapy), and clearly understand its genesis and management in conjunction with infectious diseases specialists. 


\section{Annals of Immunology \& Immunotherapy}

The diabetic patient teaches me the importance of the antibody-mediated defense against infections, by the alterations in the opsonization process, and in the antibody production, even in the phagocytosis process (with the slow \& low activity of the macrophagic system), and with the other damages consequence of the sustained high levels of the glycemia. So, when I worked at a diabetic foot facility, I insist on the need for a strictly and adequate metabolic control to increase the immune response and the activity of the antimicrobial drugs.

When I was working as Aggregated Physician in the Oncological Internal Medicine Department of a National Oncology Reference Center, I learn many things that were outside the books, I learn about the immune interactions, the complexity of the chemotherapy schemas, the importance of the NADIR (the lowest level of a blood cell count while a patient is undergoing chemotherapy), and improves my practice because I optimize the antimicrobial prophylaxis against bacteria \& fungi mainly, besides improves the use of vaccination to prevent undesirable diseases.

Actually, the use of biological therapies in many inflammatory diseases such as arthritis, psoriasis, asthma, myeloma, multiple sclerosis, etc., obligates the different medical specialties to consider the importance of immunological knowledge and put it in practice in the outpatient attention and in the hospitalization setting. A special mention should be noted in the new oncological drugs, because the use of the named "precision medicine" and the new models of agnostic medicine necessarily required an adequate immunology background in order to maximize all its potential benefits to the patients. The rise in the use of biomarkers for the diagnosis of many kinds of diseases and to select the right therapeutic options, to know the prognosis of the conditions, and to follow-up the response to treatment, shows us the importance of having a solid immunology background.

I would like to share a story that after several years was confessed to me by my students (being already several of them are specialists!), and that became an anecdote: there was a rumor that the immunology was not to my particular liking, and that I had to take the subject four times! and when I found out, I told them that I really had a fascinating immunology, and that is why I took it in the second year of medical studies, then during my Postgraduate in Medical Microbiology at Universidad Central de Venezuela, then during my Fellowship at New York University and then as part of my PhD studies! And today I can say that there is no day during medical practice, research or even reading medical papers in which I should not apply the knowledge of immunology. 